\title{
Magnetic Nanoparticles for Cancer Diagnosis and Therapy
}

\author{
Mehmet V. Yigit, Anna Moore, and Zdravka Medarova \\ Department of Radiology, Molecular Imaging Laboratory, Athinoula A. Martinos Center for \\ Biomedical Imaging, Massachusetts General Hospital Harvard Medical School, Bldg. 75, 13th St. \\ Charlestown, Massachusetts 02129, USA \\ Zdravka Medarova: zmedarova@partners.org
}

\begin{abstract}
Nanotechnology is evolving as a new field that has a potentially high research and clinical impact. Medicine, in particular, could benefit from nanotechnology, due to emerging applications for noninvasive imaging and therapy. One important nanotechnological platform that has shown promise includes the so-called iron oxide nanoparticles. With specific relevance to cancer therapy, iron oxide nanoparticle-based therapy represents an important alternative to conventional chemotherapy, radiation, or surgery. Iron oxide nanoparticles are usually composed of three main components: an iron core, a polymer coating, and functional moieties. The biodegradable iron core can be designed to be superparamagnetic. This is particularly important, if the nanoparticles are to be used as a contrast agent for noninvasive magnetic resonance imaging (MRI). Surrounding the iron core is generally a polymer coating, which not only serves as a protective layer but also is a very important component for transforming nanoparticles into biomedical nanotools for in vivo applications. Finally, different moieties attached to the coating serve as targeting macromolecules, therapeutics payloads, or additional imaging tags. Despite the development of several nanoparticles for biomedical applications, we believe that iron oxide nanoparticles are still the most promising platform that can transform nanotechnology into a conventional medical discipline.
\end{abstract}

\section{Keywords}

cancer; diagnosis; drug delivery; gene delivery; iron oxide nanoparticle; magnetic nanoparticle; molecular imaging; MRI; nanomedicine; siRNA; therapy

\section{INTRODUCTION}

According to the American Cancer Society, in the US cancer accounts for 1 out of every 4 deaths. Considering the lethality of the disease and, in most cases, the lack of symptoms until late in its progression, the best chances for survival are presented by early diagnosis. Still, the current clinical imaging methods and treatments are in many situations unable to provide timely detection and curative therapy. Chemo- and radiotherapy represent the mainstay treatments for advanced cancer. However, none of them are strictly tumor-cell specific, leading to substantial damage to healthy tissues and organs and limiting the therapeutic dose. Therefore by virtue of its capability for cell and molecular targeting, nanotechnology could provide a useful alternative to current methods $(1,2)$.

(C) Springer Science+Business Media, LLC 2012

Correspondence to: Zdravka Medarova, zmedarova@partners . org. 
Superparamagnetic iron oxide nanoparticles are a class of biomedical materials that are particularly well suited for biomedical applications. These nanoparticles are sized between 10 and $100 \mathrm{~nm}$ and are used in a wide variety of pre-clinical and clinical contexts (2-4). Specifically, their biodegradable and biocompatible nature makes them suitable for clinical studies. Currently, there are several iron oxide based materials on the market, either as MRI contrast agents or for iron supplementation (5-9).

Iron oxide nanoparticles have a superparamagnetic iron core, which makes them useful as T2 contrast agents for MRI (10-13). Since the magnetic core affects the transverse (spinspin) relaxation time of protons in nearby tissues, it creates a change (decrease) in the relaxation time reading, which can be efficiently detected by MRI (14). Such a read-out can be visualized as a darkening of the tissues on T2-weighted MR images. With this method, tissues/cells targeted by the nano-particles can be identified noninvasively. The advantages of MRI for the purposes of measuring nanoparticle uptake extend from the fact that the data collected by it are quantitative, there is a very efficient soft tissue characterization and a high tissue resolution, and the modality is tomographic with unlimited penetration into tissue (11). Therefore, being an efficient contrast agent for MRI, iron oxide nanoparticles hold significant potential both for imaging and image-guided delivery. Specifically, iron oxide nanoparticles can be detected with high sensitivity; the iron and the polymer components are biocompatible and degradable, as opposed to gadolinium (Gd), which has proven toxic effects; and the degraded iron is stored for further biological needs (11).

Around the iron core there is generally a polymer that protect the iron core from agglomeration and oxidation during the synthesis in later steps of the synthetic procedure (15). The polymer coating is important in several ways. It forms the surface of the nanoparticles and represents a versatile platform that can be modified to target specific biological compartments, e.g. blood pool, lymph nodes, liver, etc. For example, polyethylene glycol coated iron oxide nanoparticles are long-circulating due to their minimized adsorbtion by blood proteins or other biological components in the body (16). The coating is also important in serving as a template for targeting macromolecules, imaging tags and delivering therapeutic payloads (Fig. 1a) (17). The targeting macromolecules could be small peptides or functional nucleic acids, which bind to surface antigens overexpressed by certain cell types. These moieties increase the uptake of the nanoparticles by cancer cells and produce tumor-specific contrast or increase the therapeutic payload to these cells. In order to obtain the capability for detection by an additional imaging modality, the coating can be functionalized with near infrared fluorescent dyes for in vivo or ex vivo fluorescent imaging (18-20), Gd chelates to provide T1-weighted MR contrast (21), positron emission tomography (PET) tracers for MRI/PET dual imaging (22), or gold nanoparticles for MRI/ surface enhanced Raman scattering (SERS) imaging (23). Finally, the therapeutic payload could be a small molecule drug, such as doxorubicin (24-26); an oligonucleotide, such as small interfering RNAs (27-29); or a pro-apoptotic peptide (30), such as RGD.

As the imaging potential of iron oxide nanoparticles is already reported numerous times and very well accepted, in this review we will focus on the combined delivery and imaging applications which involve iron oxide nanoparticles complexed to therapeutic moieties.

\section{SYNTHESIS OF IRON OXIDE NANOPARTICLES AND SUPERPARAMAGNETIC FEATURE}

Synthesis of iron oxide nanoparticles has been achieved via physical and chemical methods $(31,32)$. Owing to the higher level of control over the size, composition, magnetic properties and shapes of the nanoparticles, wet chemistry procedures have been investigated more extensively than physical methods $(3,33)$. Iron oxides are generally prepared by co- 
precipitation of $\mathrm{Fe}^{2+}$ and $\mathrm{Fe}^{3+}$ salts instead of $\mathrm{Co}$ or $\mathrm{Ni}$ in an aqueous solution due to the less toxic effect of the resulting iron oxides (34). The resulting core consists of magnetite $\left(\mathrm{Fe}_{3} \mathrm{O}_{4}\right)$, maghemite $\left(\gamma-\mathrm{Fe}_{2} \mathrm{O}_{3}\right)$ or a mixture of the two. The anionic salt content (chlorides, nitrates, sulphates etc.), the $\mathrm{Fe}^{2+}$ and $\mathrm{Fe}^{3+}$ ratio, $\mathrm{pH}$ and the ionic strength in the aqueous solution all play a role in controlling the size (12). It is important to prevent the oxidation of the synthesized nanoparticles and protect their magnetic properties by carrying out the reaction in an oxygen free environment under inert gas such as nitrogen or argon (35). The coating materials are generally added during the co-precipitation process in order to prevent the agglomeration of the iron oxides into microparticles $(15,16)$. There have been several surface coating materials used for stabilizing iron oxide nanoparticles, among which are synthetic and natural polymers, such as polyethylene glycol (PEG), dextran, polyvinylpyrrolidone (PVP), fatty acids, polypeptides, chitosin, gelatin, etc. $(3,36)$. The choice of the coating is essential for transforming the nanoparticles into clinical materials and controlling their size.

Superparamagnetism is an important feature of iron oxide nanoparticles since it permits their detection by MRI (37-39). For superparamagnetism to take effect, each individual nanoparticle core has to be small enough to have no measurable permanent dipole moment in the absence of an external magnetic field $(40,41)$. On the other hand, a nanoparticle suspension has a much greater magnetic susceptibility than paramagnetic materials and is greatly magnetized by an external magnetic field (Fig. 1b) $(42,43)$.

The superparamagnetic behavior of magnetic nanoparticles is not only a core size effect but may also depend on surface modifications. The nanoparticles should be properly coated to reduce magnetic dipole-dipole interactions $(44,45)$.

\section{IMAGE-GUIDED CANCER THERAPY}

Cancer diagnosis by nanotechnology is an emerging field. Detecting cancer noninvasively (a feature of nanotechnology) benefits the patient in obvious and subtle ways. For instance, noninvasive imaging could obviate the need for tissue sampling through biopsy and thus reduce the physical burden on the patient. Noninvasive imaging of cancer with high sensitivity is crucial and achievable, as an inherent property of magnetic nanoparticles. Moreover, a slight modification of the nanoparticle to include a therapeutic moiety offers the possibility of combining diagnosis with an initial drug delivery step.

Image-guided therapy is currently the most active research area in applications of magnetic nanoparticle technology to biomedicine. As new cancer markers are discovered, genes responsible for the progression, proliferation, invasiveness and drug resistance of cancer will be identified. Discovery of therapeutics for cancer will become a major focus of drug companies.

While it is crucial to design the drugs, imaging the drug's in vivo bioavailability, function and therapeutic potential in real time is important for the best drug discovery outcome. Moreover, magnetic nanoparticle technology has demonstrated the possibility of combining diagnosis with therapy, in a single step, in medical situations where it would pose a distinct advantage.

There are several methods reported in the literature that suggest how therapy can be combined with imaging (diagnosis): incorporating the therapeutic materials into polymer coating by covalent or non-covalent chemical binding; hydrophobic or electrostatic interaction; or encapsulating the drug molecules $(24,26,46-49)$. Covalent incorporation is generally achieved through a bifunctional linker, which binds to the nanoparticle at one end and to the drug molecule at the other end (46). The therapeutic group could be genetic 
material such as a small interfering RNA (siRNA) or a small drug molecule such as doxorubicin or paclitaxel. In this review, nanoparticle administration by two means (intravenously injected and cell labeling) will be discussed for combined therapy and imaging.

\section{INTRAVENOUSLY ADMINISTERED NANOPARTICLES}

Intravenous injection is a direct way to administer the probes into the blood stream of the animal. In most cases this is the fastest way to introduce the compounds to the whole body. Therapeutic nanoparticles in general are administered using this method. This way the nanoparticles circulate in the body and reach multiple tissues at the same time. This is important as multiple tumor sites can be targeted with one injection. Here, we will discuss image-guided therapy that involves intravenous injection under two categories.

\section{Genetic Material Conjugated Magnetic Nanoparticles}

Ever since RNA interference (RNAi) was discovered in Caenorhabditis elegans in 1998, using RNAi to address questions in human disease has proceeded unabated. RNAi based drug complexes became the focus of many scientists attempting to cure disease at the mRNA level. The strategy for using RNAi in human diseases relies on identifying the sequence of the gene responsible for the disease and administering a complementary short synthetic double stranded RNA that targets and binds the identified gene, inhibiting its function. As a result, disease related protein expression is brought under control, its progression is inhibited and the disease is arrested at the cellular level.

Auxilliary questions as to the successful use of RNAi include how to preferentially shuttle the siRNA molecules to diseased cells, to increase their bioavailability in vivo and to prevent their degradation by nucleases. To overcome these challenges robust delivery vehicles are needed.

Iron oxide nanoparticles have great potential as such, because it is possible to direct the nanoparticles to diseased tissues by having targeting groups on the nanoparticle. Moreover, it is possible to load several molecules on the polymer coating of an individual nanoparticle. Iron oxide nanoparticles are also advantageous because of their capability of detection by noninvasive imaging. This allows one to track siRNA bioavailability by MRI or in vivo optical imaging (if a fluorescent probe has been attached to each nanoparticle). It has been also shown that the nanoparticles protect the siRNA molecules from degradation (50).

SiRNA functionalized iron oxide nanoparticles have been used to address several potential medical applications. In one of the early studies, iron oxide nanoparticles were complexed with siRNA to knockdown the expression of green fluorescent protein (GFP) in mice implanted with GFP expressing tumor cells (47). In this proof of principle study, the amine terminals of the iron oxide nanoparticles were functionalized with a bifunctional linker, which was then coupled to $5^{\prime}$ thiol-modified short siRNA molecules targeting specifically the gene responsible for GFP expression, Fig. 2.

In order to elevate their uptake, the nanoparticles were further functionalized with arginine rich cationic peptide (MPAP), which facilitate nanoparticle uptake by electrostatic interaction between the positively charged peptide and the negatively charged cell membrane. The nanoparticles were also conjugated to an NIR fluorescent probe (Cy5.5) so that it would be possible to measure the uptake of particles by both in vivo optical imaging and in vivo MRI. 
In a more medically relevant study, nanoparticles were coupled to siRNA to knockdown the anti-apoptotic gene birc5 that encodes Survivin, a member of the apoptotic protein inhibitor family. Survivin is overexpressed by most human cancers. After administration of the probe, there was a significant increase in the rate of apoptosis among treated tumor cells due to downregulation of birc 5 expression.

A recent study showed that if nanoparticles were conjugated to a more target-specific peptide, EPPT, (rather than polyarginine peptide (MPAP)) tumoral uptake increased and an improved silencing effect was observed (46). In these studies the tumor progression, nanoparticle uptake by tumor and bioavailabilty of the nanoparticle complex were validated and semi-quantified by in vivo noninvasive MRI and optical microscopy. Other studies have been carried out, targeting different genes and cancer markers (51).

In another study Zhang and coworkers established a clorotoxin-labeled magnetic nanomaterial for targeted gene delivery to glioma. In this proof of principle study the nanomaterial consisted of a magnetic nanoparticle core coated by a copolymer of chitosan, polyethylene glycol, and polyethylenimine (52). The nanomaterial was conjugated to GFP encoding DNA for genetic alteration in glioma cells and chlorotoxin for specific accumulation. The nanoparticles were intravenously injected to mice bearing glioma xenografts. The results showed a higher GFP expression and nanoparticle accumulation in implanted glioma cells, which was evaluated by in vivo MRI and optical imaging as compared to mice injected with nanoparticles not labeled with DNA or chlorotoxin. This proof of principle study can be later used for targeting a disease related gene in glioma cells.

In a different study Bhujwalla and coworkers encapsulated clinically relevant iron oxide nanoparticles (Feridex or Magnevist) and cyclooxygenase-2 (COX-2) specific siRNA; an important therapeutic target in breast cancer; into PEGylated liposomes (53). Intravenous administration of the PEGylated liposomes showed accumulation of the nanoparticles in the tumor. Downregulation of COX-2 was observed in cultured cells treated with the liposomes.

In another study Bhatia and coworkers synthesized a magnetofluorescent nanomaterial conjugated to dendrimers (dendriworms) for delivery of siRNA to human glioblastoma cells. The siRNA targeted to the epidermal growth factor receptor (EGFR) was conjugated to dendriworms and administered to mice bearing an EGFR-driven model of glioblastoma. The results showed significant downregulation of EGFR protein in human glioblastoma cells by $70-80 \%$. This effect was 2.5 -fold more efficient than commercial cationic lipids, presumably extending from the specific design of the dendriworms, optimized for a maximal endosomal escape (54). The authors suggested that the enrichment of polymers along the nanoworm backbone improved endosomal escape through the proton sponge effect, resulting in more effective silencing.

\section{Drug Encapsulated Magnetic Nanoparticles}

Chemotherapy is one major therapeutic approach to fighting cancer. The flaw in this approach is the lack of specificity for cancer cells and the high accompanying toxicity. It is crucial to migrate chemotherapy into a safer and more efficient therapy. To do so, decreasing the dosage is important. This can be achieved by delivering a sufficient amount of drug molecules into diseased tissues, while preventing or decreasing the amount taken up by healthy cells, which can be achieved by cell-specific chemotherapy delivery routes.

In a study done by Haam and coworkers, magnetic nanocrystals and chemotherapeutic drug molecules (DOX) were encapsulated by amphiphilic block copolymers referred to as multifunctional magnetopolymeric nanohybrids (MMPNs) (55). The MMPNs were used to image breast cancer tumors by targeting the overexpressed HER2/neu cancer marker in vitro 
and in vivo. The targeting was achieved by incorporating anti-HER antibody (HER, herceptin) on MMPNs. Results showed that when the targeting and the drug loading combined in MMNP, a significantly higher tumor growth inhibition is observed in mice implanted with breast cancer xenografts.

In a different study Jon and coworkers synthesized super-paramagnetic iron oxide nanoparticles coated with negatively charged anti-biofouling polymer and attached cy5.5 (NIR dye) on the surface (56). The nanoparticles were then loaded with positively charged doxorubicin (DOX) using electrostatic interaction. The nanoparticles were systematically injected into lung cancer bearing mice resulting in a significant decrease in tumor size. However when the mice were injected with nanoparticles not loaded with DOX, DOX alone, or an eight-fold higher dose of DOX, no similar effect was observed. The nanoparticle uptake in this study was achieved by the enhanced permeability (EPR) and retention effect using a passive targeting strategy. The nanoparticles were mostly taken up by tumors and the liver, whereas lung uptake was diminished by injecting the nanoparticles in a solution of $5 \%$ glucose rather than PBS. The tumoral uptake was evaluated by in vivo MRI and ex vivo optical imaging.

In other work by Lim et al. drug molecules bound to magnetic nanoparticles were activated specifically in cancer cells by taking advantage of the $\mathrm{pH}$ differences between the intracellular conditions of malignant and non-malignant cells (57). The authors used magnetic nanoparticles to shuttle doxorubicin drug molecules specifically to breast cancer tumor sites and activate them in the tumor environment, therefore achieving a more target specific treatment for breast cancer, while imaging the therapeutic outcome by noninvasive MRI.

The authors synthesized nanoparticles with a $\mathrm{MnFe}_{2} \mathrm{O}_{4}$ magnetic core detectable by $\mathrm{T} 2$ weighted MRI. The nanoparticles were coated with polyethylene glycol (PEG) derivatives, which have been traditionally used to coat nanoparticles. The polymer coating encapsulated the doxorubicin drug molecules by means of the $\pi-\pi$ interaction between the pyrene groups of the polymer coating and the doxorubicin. The nanoparticles released the doxorubicin load in the protein rich intracellular acidic environments of cancer cells by protonation of doxorubicin and weakening of the $\pi-\pi$ interaction. On the other hand, in the neutral intracellular environment of non-malignant cells drug release was slow and the effect of the drug molecule was decreased. In order to have the system more specific to breast cancer (Her2/neu overexpressed) the nanoparticles were conjugated with anti-HER/neu antibody, Fig. 3. The system is advantageous not only because there is a cell specific delivery and drug release but also because the drug administration can be adjusted by defining its bioavailability in tumors monitored by in vivo MRI.

In a similar study the magnetic nanoparticles were functionalized with an RNA aptamer that is specific to prostate specific membrane antigen (PSMA) overexpressed in $\mathrm{LnCaP}$ prostate cancer cells (58). The doxorubicin molecules were intercalated in the RNA strands attached to the nanoparticles. Upon uptake of the nanoparticles by the cancer cells, the nanoparticles released the intercalated doxorubicin molecules and killed the cells. The tumors of the mice implanted with $\mathrm{LNCaP}$ prostate cancer cells had a greater nanoparticle accumulation and slower tumor growth when injected with the experimental nanoparticle.

\section{MAGNETIC NANOPARTICLES FOR IMAGE-GUIDED CELL TRACKING}

There are numerous examples of cellular therapies in preclinical studies. However most of them cannot be translated into a clinical setting due to several issues (59). One big obstacle is the lack of knowledge about the bioavailability of the injected cells in vivo. This key point can be addressed by noninvasive imaging. There are several methods to track cells 
noninvasively. Among these are scintigraphy, single photon emission computed tomography (SPECT), and positron emission tomography (PET) (59). MRI provides excellent soft-tissue contrast and multidimensional functional, structural and morphological information but it suffers from low-sensitivity (60). Optical imaging provides high sensitivity and real-time tracking of biological processes. However it suffers from limited deep-tissue penetration and background autofluorescence (60). SPECT and PET have high-sensitivity in tracking biological events but they have a poor spatial resolution $(60,61)$. Therefore multimodal imaging methods that combine several imaging techniques are explored for preclinical and clinical studies. Here, we are going to only focus on examples that involve magnetic nanoparticle-enhanced MRI.

In an early example authors showed that it is possible to track the fate of injected dendritic cells for cellular cancer therapy using MRI (62). In this example, in vitro generated dendritic cells loaded with tumor-derived antigenic peptides were labeled with clinically safe ultra small iron oxide nanoparticles (Endorem) for MRI and with ${ }^{111}$ Inoxine for scintigraphy. The cells naturally took up substantial amounts of the nanoparticles by endocytosis without functional impairment. The cells were injected into the lymph nodes of stage-III melanoma patients and were imaged by noninvasive MRI, Fig. 4. By this methodology it was possible to quantify the percentage of cells migrating to lymph nodes away from the injected site using high resolution MRI. MRI was superior to scintigraphic imaging in terms of resolution, identifying the lymph nodes to which the cells migrated, and quantifying the number of cells localized in the injected lymph node or other distant nodes.

Another question that has been addressed using magnetic nanoparticle-enhanced MRI is the extent of the immune response against cell-based vaccines. Monitoring the antigen capture by antigen-presenting cells (APCs) and its delivery to lymph nodes has been a challenge. In response, Long et al. labeled B16 tumor cells with magnetic nanoparticles, which were then injected into mice as a vaccine after irradiation (63). The cancer cells carrying the magnetic nanoparticles were captured by APCs, leading to internalization, APC labeling and trafficking to lymph nodes in vivo. This cellular event was imaged by high resolution in vivo MRI. In this case, MRI helped to track the antigen in vivo as a correlate to therapeutic efficacy.

\section{CONCLUSIONS AND FUTURE PERSPECTIVES}

Magnetic nanoparticles have been used extensively and successfully as T2-weighted MRI contrast agents, drug delivery vehicles, magnetic sensors or hyperthermia-generating probes. Although there is agreement on the potential that these nanoparticles hold, there are still key issues that need to be resolved before clinical translation of these particles. One issue is the need to have better control over the size and magnetization of the nanoparticles. Having reproducible particle sizes with narrow size distributions, and simpler synthetic routes that generate a large scale, cost-effective products are important prerequisites for clinical translation.

It is also crucial to address any toxicity associated with with administration of iron oxide nanoparticles. There are several potentially toxic effects associated with exposure to iron oxide nanoparticles. For instance, inflammation, the formation of apoptotic bodies, impaired mitochondrial function, membrane leakage of lactate dehydrogenase, generation of reactive oxygen species, chromosomal damage and chromosome condensation are some of these reported effects (64). Therefore, since there is a significant effort in using iron oxide nanoparticles in clinical studies for both diagnosis and therapy, the biocompatibility and biosafety concerns associated with their usage should be thoroughly addressed. 
Another important issue that needs to be addressed is nanoparticle bioavailability. It is crucial to maximize the interaction of the nanoparticles with the target tissues and to eliminate or minimize the uptake by other organs. There is a significant effort in that direction. It is also necessary to have particles with greater magnetic susceptibility in order to increase the sensitivity of detection by MRI. The vast majority of the current studies involving the application of magnetic nanoparticles to cancer biology are at the research stage. Still, provided that solutions to the above issues are found, we believe that routine clinical application of magnetic nanoparticles is likely in the near future.

\section{ABBREVIATIONS}

$\begin{array}{ll}\text { APC } & \text { antigen-presenting cell } \\ \text { DMNP } & \text { drug-delivering magnetic nanoparticles } \\ \text { DOX } & \text { doxorubicin } \\ \text { Gd } & \text { gadolinium } \\ \text { GFP } & \text { green fluorescent protein } \\ \text { HER } & \text { anti HER2/neu antibody } \\ \text { IRR } & \text { irrelevant antibody } \\ \text { MN } & \text { magnetic nanoparticle } \\ \text { NIR } & \text { near infrared } \\ \text { RFP } & \text { red fluorescent protein } \\ \text { R2 } & \text { transverse relaxation rate } \\ \text { T1 } & \text { longitudinal relaxation time } \\ \text { T2 } & \text { transverse relaxation time }\end{array}$

\section{References}

1. Pautler M, Brenner S. Nanomedicine: promises and challenges for the future of public health. Int J Nanomedicine. 2010; 5:803-9. [PubMed: 21042425]

2. Kievit FM, Zhang M. Surface engineering of iron oxide nanoparticles for targeted cancer therapy. Acc Chem Res. 2011; 44:853-62. [PubMed: 21528865]

3. Gupta AK, Wells S. Surface-modified superparamagnetic nanoparticles for drug delivery: preparation, characterization, and cytotoxicity studies. IEEE Trans NanoBiosci. 2004; 3:66-73.

4. Pankhurst QA, Connolly J, Jones SK, Dobson J. Applications of magnetic nanoparticles in biomedicine. J Phys D: Appl Phys. 2003; 36:R167-81.

5. Anzai Y, Piccoli CW, Outwater EK, et al. Evaluation of neck and body metastases to nodes with ferumoxtran 10-enhanced MR imaging: phase III safety and efficacy study. Radiology. 2003; 228:777-88. [PubMed: 12954896]

6. Kim HS, Oh SY, Joo HJ, Son KR, Song IC, Moon WK. The effects of clinically used MRI contrast agents on the biological properties of human mesenchymal stem cells. NMR Biomed. 2010; 23:514-22. [PubMed: 20175151]

7. Provenzano R, Schiller B, Rao M, Coyne D, Brenner L, Pereira BJG. Ferumoxytol as an intravenous iron replacement therapy in hemodialysis patients. Clin J Am Soc Nephrol. 2009; 4:386-93. [PubMed: 19176796]

8. Saokar A, Gee MS, Islam T, Mueller PR, Harisinghani MG. Appearance of primary lymphoid malignancies on lymphotropic nanoparticle-enhanced magnetic resonance imaging using ferumoxtran-10. Clin Imag. 2010; 34:448-52. 
9. Spinowitz BS, Kausz AT, Baptista J, et al. Ferumoxytol for treating iron deficiency anemia in CKD. J Am Soc Nephrol. 2008; 19:1599-605. [PubMed: 18525001]

10. Babes L, Denizot B, Tanguy G, Le JJJ, Jallet P. Synthesis of iron oxide nanoparticles used as MRI contrast agents: a parametric study. J Colloid Interface Sci. 1999; 212:474-82. [PubMed: 10092379]

11. Bulte JW, Kraitchman DL. Iron oxide MR contrast agents for molecular and cellular imaging. NMR Biomed. 2004; 17:484-99. [PubMed: 15526347]

12. Sjogren CE, Briley-Saebo K, Hanson M, Johansson C. Magnetic characterization of iron oxides for magnetic resonance imaging. Magn Reson Med. 1994; 31:268-72. [PubMed: 8057797]

13. Weissleder R, Bogdanov A, Neuwelt EA, Papisov M. Long-circulating iron oxides for MR imaging. Adv Drug Delivery Rev. 1995; 16:321-33.

14. Josephson L, Lewis J, Jacobs P, Hahn PF, Stark DD. The effects of iron oxides on proton relaxivity. Magn Reson Imag. 1988; 6:647-53.

15. Gupta AK, Gupta M. Synthesis and surface engineering of iron oxide nanoparticles for biomedical applications. Biomaterials. 2005; 26:3995-4021. [PubMed: 15626447]

16. Sun C, Du K, Fang C, et al. PEG-Mediated synthesis of highly dispersive multifunctional superparamagnetic nanoparticles: their physicochemical properties and function in vivo. ACS Nano. 2010; 4:2402-10. [PubMed: 20232826]

17. Zhang Y, Kohler N, Zhang M. Surface modification of superparamagnetic magnetite nanoparticles and their intracellular uptake. Biomaterials. 2002; 23:1553-61. [PubMed: 11922461]

18. Corsi F, De Palma C, Colombo M, et al. Towards ideal magnetofluorescent nanoparticles for bimodal detection of breast-cancer cells. Small. 2009; 5:2555-64. [PubMed: 19634132]

19. Pittet MJ, Swirski FK, Reynolds F, Josephson L, Weissleder R. Labeling of immune cells for in vivo imaging using magnetofluorescent nanoparticles. Nat Protoc. 2006; 1:73-9. [PubMed: 17406214]

20. Wang P, Yigit MV, Medarova Z, et al. Combined small interfering RNA therapy and in vivo magnetic resonance imaging in islet transplantation. Diabetes. 2011; 60:565-71. [PubMed: 21270267]

21. Bae KH, Kim YB, Lee Y, Hwang J, Park H, Park TG. Bioinspired synthesis and characterization of gadolinium-labeled magnetite nanoparticles for dual contrast T1- and T2-weighted magnetic resonance imaging. Bioconjugate Chem. 2010; 21:505-12.

22. Yang X, Hong H, Grailer JJ, et al. cRGD-functionalized, DOX-conjugated, and Cu-labeled superparamagnetic iron oxide nanoparticles for targeted anticancer drug delivery and PET/MR imaging. Biomaterials. 2011; 32:4151-60. [PubMed: 21367450]

23. Yigit MV, Zhu L, Ifediba MA, et al. Noninvasive MRI-SERS imaging in living mice using an innately bimodal nanomaterial. ACS Nano. 2011; 5:1056-66. [PubMed: 21194236]

24. Chen FH, Zhang LM, Chen QT, Zhang Y, Zhang ZJ. Synthesis of a novel magnetic drug delivery system composed of doxorubicin-conjugated $\mathrm{Fe} 3 \mathrm{O} 4$ nanoparticle cores and a PEG-functionalized porous silica shell. Chem Commun (Camb). 2010; 46:8633-5. [PubMed: 20941412]

25. Gaihre B, Khil MS, Kim HY. In vitro anticancer activity of doxorubicin-loaded gelatin-coated magnetic iron oxide nanoparticles. J Microencapsul. 2011; 28:286-93. [PubMed: 21545319]

26. Kievit FM, Wang FY, Fang C, et al. Doxorubicin loaded iron oxide nanoparticles overcome multidrug resistance in cancer in vitro. J Contr Release. 2011; 152:76-83.

27. Lee JH, Lee K, Moon SH, Lee Y, Park TG, Cheon J. All-in-one target-cell-specific magnetic nanoparticles for simultaneous molecular imaging and siRNA delivery. Angew Chem Int Ed. 2009; 48:4174-9.

28. Taratula O, Garbuzenko O, Savla R, Wang YA, He H, Minko T. Multifunctional nanomedicine platform for cancer specific delivery of siRNA by superparamagnetic iron oxide nanoparticlesdendrimer complexes. Curr Drug Deliv. 2011; 8:59-69. [PubMed: 21034421]

29. Zhang H, Lee MY, Hogg MG, Dordick JS, Sharfstein ST. Gene delivery in three-dimensional cell cultures by superparamagnetic nanoparticles. ACS Nano. 2010; 4:4733-43. [PubMed: 20731451]

30. Ellerby HM, Arap W, Ellerby LM, et al. Anti-cancer activity of targeted pro-apoptotic peptides. Nat Med. 1999; 5:1032-8. [PubMed: 10470080] 
31. Lee CS, Lee H, Westervelt RM. Microelectromagnets for the control of magnetic nanoparticles. Appl Phys Lett. 2001; 79:3308-10.

32. Stolnik S, Illum L, Davis SS. Long circulating microparticulate drug carriers. Adv Drug Deliv Rev. 1995; 16:195-214.

33. Gupta AK, Curtis ASG. Lactoferrin and ceruloplasmin derivatized superparamagnetic iron oxide nanoparticles for targeting cell surface receptors. Biomaterials. 2004; 25:3029-40. [PubMed: 14967536]

34. Tourinho F, Franck R, Massart R, Perzynski R. Synthesis and magnetic properties of manganese and cobalt ferrite ferrofluids. Prog Colloid Polym Sci. 1989; 79:128-34.

35. Kim DK, Zhang Y, Voit W, Rao KV, Muhammed M. Synthesis and characterization of surfactantcoated superparamagnetic monodispersed iron oxide nanoparticles. J Magn Magn Mater. 2001; 225:30-6.

36. Portet D, Denizot B, Rump E, Lejeune J-J, Jallet P. Nonpolymeric coatings of iron oxide colloids for biological use as magnetic resonance imaging contrast agents. J Colloid Interface Sci. 2001; 238:37-42. [PubMed: 11350133]

37. Bonnemain B. Superparamagnetic agents in magnetic resonance imaging. Physicochemical characteristics and clinical applications. A review. J Drug Target. 1998; 6:167-74. [PubMed: 9888302]

38. Kim DK, Zhang Y, Voit W, et al. Superparamagnetic iron oxide nanoparticles for biomedical applications. Scr Mater. 2001; 44:1713-7.

39. Wang YX, Hussain SM, Krestin GP. Superparamagnetic iron oxide contrast agents: physicochemical characteristics and applications in MR imaging. Eur Radiol. 2001; 11:2319-31. [PubMed: 11702180]

40. Bean CP, Livingston JD. Superparamagnetism. J Appl Phys. 1959; 30:120S-9S.

41. Tartaj P, Morales MdP, Veintemillas-Verdaguer S, Gonzalez-Carreno T, Serna CJ. The preparation of magnetic nanoparticles for applications in biomedicine. J Phys D: Appl Phys. 2003; 36:R18297.

42. Goya GF, Berquó TS, Fonseca FC, Morales MP. Static and dynamic magnetic properties of spherical magnetite nanoparticles. J Appl Phys. 2003; 94:3520-8.

43. Jeong U, Teng X, Wang Y, Yang H, Xia Y. Superparamagnetic colloids: controlled synthesis and niche applications. Adv Mater. 2007; 19:33-60.

44. Mikhaylova M, Kim DK, Bobrysheva N, et al. Superparamagnetism of magnetite nanoparticles: dependence on surface modification. Langmuir. 2004; 20:2472-7. [PubMed: 15835712]

45. Bedanta S, Kleemann W. Supermagnetism. J Phys D: Appl Phys. 2009; 42:013001.

46. Kumar M, Yigit M, Dai G, Moore A, Medarova Z. Image-guided breast tumor therapy using a small interfering RNA nanodrug. Cancer Res. 2010; 70:7553-61. [PubMed: 20702603]

47. Medarova Z, Pham W, Farrar C, Petkova V, Moore A. In-vivo imaging of siRNA delivery and silencing in tumors. Nat Med. 2007; 13:372-7. [PubMed: 17322898]

48. Medarova Z, Rashkovetsky L, Pantazopoulos P, Moore A. Multi-parametric monitoring of tumor response to chemotherapy by noninvasive imaging. Cancer Res. 2009; 69:1182-9. [PubMed: 19141648]

49. Sun C, Lee JS, Zhang M. Magnetic nanoparticles in MR imaging and drug delivery. Adv Drug Deliv Rev. 2008; 60:1252-65. [PubMed: 18558452]

50. Ferrari M. Vectoring siRNA therapeutics into the clinic. Nat Rev Clin Oncol. 2010; 7:485-6. [PubMed: 20798696]

51. Bartlett DW, Su H, Hildebrandt IJ, Weber WA, Davis ME. Impact of tumor-specific targeting on the biodistribution and efficacy of siRNA nanoparticles measured by multimodality in vivo imaging. Proc Natl Acad Sci U S A. 2007; 104:15549-54. [PubMed: 17875985]

52. Kievit FM, Veiseh O, Fang C, et al. Chlorotoxin labeled magnetic nanovectors for targeted gene delivery to glioma. ACS Nano. 2010; 4:4587-94. [PubMed: 20731441]

53. Mikhaylova M, Stasinopoulos I, Kato Y, Artemov D, Bhujwalla ZM. Imaging of cationic multifunctional liposome-mediated delivery of COX-2 siRNA. Cancer Gene Ther. 2009; 16:21726. [PubMed: 18927599] 
54. Agrawal A, Min DH, Singh N, et al. Functional delivery of siRNA in mice using dendriworms. ACS Nano. 2009; 3:2495-504. [PubMed: 19673534]

55. Yang J, Lee $\mathrm{CH}$, Ko HJ, et al. Multifunctional magneto-polymeric nanohybrids for targeted detection and synergistic therapeutic effects on breast cancer. Angew Chem Int Ed Engl. 2007; 46:8836-9. [PubMed: 17943947]

56. Yu MK, Jeong YY, Park J, et al. Drug-loaded superparamagnetic iron oxide nanoparticles for combined cancer imaging and therapy in vivo. Angew Chem Int Ed Engl. 2008; 47:5362-5. [PubMed: 18551493]

57. Lim E-K, Huh Y-M, Yang J, Lee K, Suh J-S, Haam S. pH-Triggered drug-releasing magnetic nanoparticles for cancer therapy guided by molecular imaging by MRI. Adv Mater. 2011; 23:2436-42. [PubMed: 21491515]

58. Yu MK, Kim D, Lee I-H, So J-S, Jeong YY, Jon S. Image-guided prostate cancer therapy using aptamer-functionalized thermally cross-linked superparamagnetic iron oxide nanoparticles. Small. 2011; 7:2241-9. [PubMed: 21648076]

59. Srinivas M, Aarntzen EH, Bulte JW, et al. Imaging of cellular therapies. Adv Drug Deliv Rev. 2010; 62:1080-93. [PubMed: 20800081]

60. Roach M 3rd, Alberini JL, Pecking AP, et al. Diagnostic and therapeutic imaging for cancer: therapeutic considerations and future directions. J Surg Oncol. 2011; 103:587-601. [PubMed: 21480253]

61. Spencer SS, Theodore WH, Berkovic SF. Clinical applications: MRI, SPECT, and PET. Magn Reson Imag. 1995; 13:1119-24.

62. de Vries IJ, Lesterhuis WJ, Barentsz JO, et al. Magnetic resonance tracking of dendritic cells in melanoma patients for monitoring of cellular therapy. Nat Biotechnol. 2005; 23:1407-13.

[PubMed: 16258544]

63. Long CM, van Laarhoven HW, Bulte JW, Levitsky HI. Magneto-vaccination as a novel method to assess and quantify dendritic cell tumor antigen capture and delivery to lymph nodes. Cancer Res. 2009; 69:3180-7. [PubMed: 19276358]

64. Singh N, Jenkins GJ, Asadi R, Doak SH. Potential toxicity of super-paramagnetic iron oxide nanoparticles (SPION). Nano Rev. 2010; 1:5358.10.3402/nano.v1i0.5358 


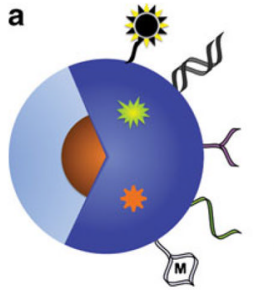

Iron Oxide Core

Coating

Radiolabel

澊 NIR dye

10. Gd complex

$\prec$ Targeting peptide

$\sim$ Targeting ligand

* Drug molecule

b

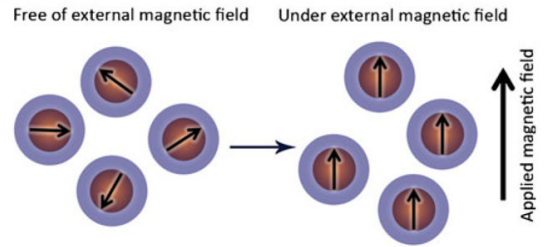

Fig. 1.

(a) The magnetic nanoparticle is composed of magnetic core/cores around which there is a coating. The coating is complexed with different moieties for additional functionalities. (b) Superparamagnetic iron oxide nanoparticles have a single magnetic domain because of their small magnetic core and are greatly magnetized under an externally applied magnetic field. 

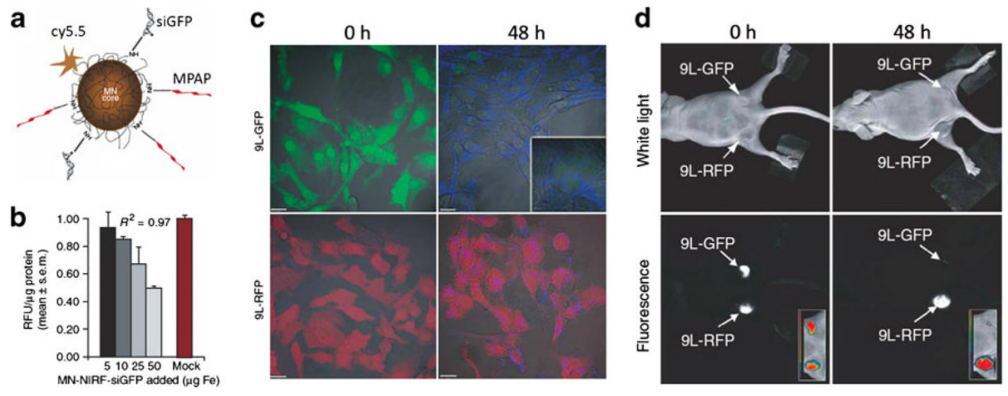

Fig. 2.

(a) Magnetic nanoparticles functionalized with siRNA targeting GFP expression (MNNIRF-siGFP). (b) In vitro testing of MN-NIRF-siGFP cell uptake and silencing efficiency in stably transfected 9 L-GFP gliosarcoma cells. (b) The silencing effect was manifested as a concentration-dependent decrease in GFP relative fluorescence levels. Data represent an average of three experiments. RFU, relative fluorescence units. c Confocal microscopy showing MN-NIRF-siGFP accumulation in 9 L-GFP and 9 L-RFP cells. Note that although the probe accumulated in both cell lines (Cy5.5 fluorescence, blue), there was a substantial silencing effect in the 9 L-GFP cell line, resulting in marked suppression of GFP fluorescence. Scale bar, $20 \mu \mathrm{m}$. (d) In vivo imaging of MN-NIRF-siGFP silencing in tumors. (a) In vivo NIRF optical imaging of mice bearing bilateral 9 L-GFP and 9 L-RFP tumors $48 \mathrm{~h}$ after intravenous probe injection. There was a marked decrease in $9 \mathrm{~L}-\mathrm{GFP}-$ associated fluorescence $(P=0.0083)$ and no change in 9 L-RFP fluorescence. To generate GFP/RFP reconstructions, GFP and RFP images were acquired separately and then merged. (Reprinted with permission from Ref 47 by Nature Publishing Group). 


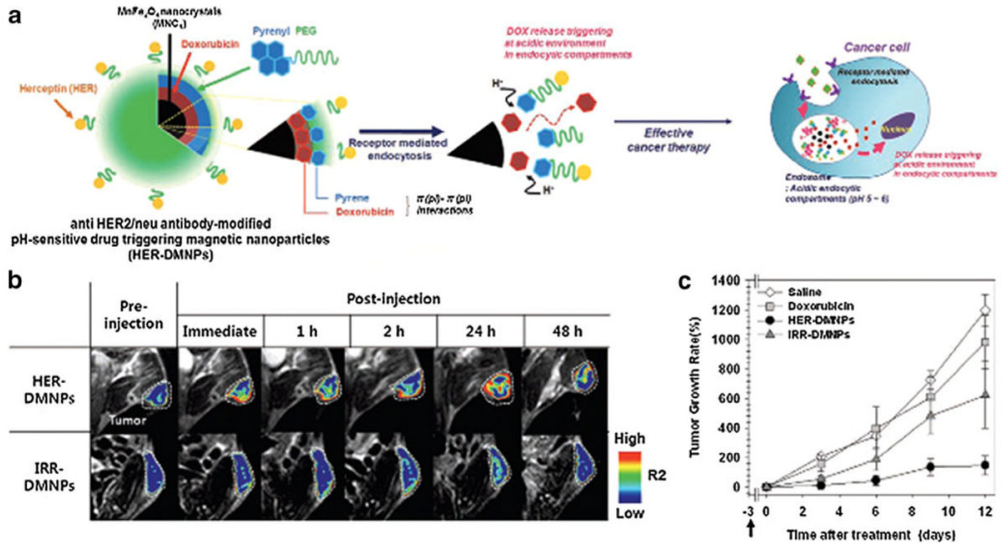

Fig. 3.

(a) Schematic illustration of anti HER2/neu antibody-modified $\mathrm{pH}$-sensitive drug-releasing magnetic nanoparticles (HER-DMNPs) for cancer therapy followed by MRI. (b) Colorcoded T 2-weighted MR images of tumor-bearing mice after the intravenous injection of HER-DMNPs and IRR-DMNPs at various time intervals, respectively. Tumor regions are indicated with a white dashed boundary. (c) Comparative therapeutic efficacy study in the in vivo model. (black circle: HER-DMNPs, dark gray triangle: IRR-DMNPs, gray square: DOX, and white diamond: saline). Black arrow indicates the day of cancer cells (NIH3T6.7 cells) implantation in mice. (Reprinted with permission from Ref 57 by John Wiley and Sons.) 


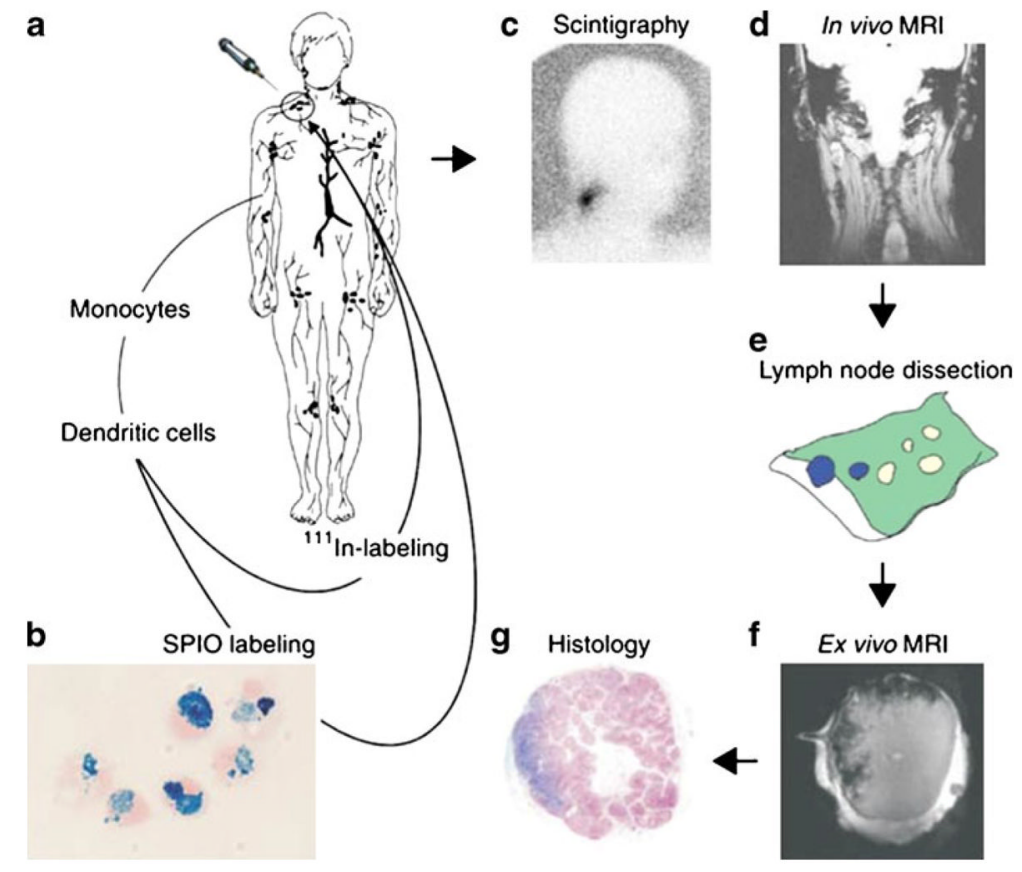

Fig. 4.

(a) Monocytes are obtained by cytopheresis from stage-III melanoma patients. (b) They are cultured and labeled with SPIO particles and 111In. (c,d) The cells are then injected intranodally into a (either cervical, inguinal or axillary) lymph node basin that is to be resected and their biodistribution is monitored in vivo by scintigraphy (c) and MRI at 3 Tesla (d). (e-g) The lymph node basis is resected and separate lymph nodes are visualized with high resolution MRI at 7 Tesla (f) and histology (g). (Reprinted with permission from Ref 62 by Nature Publishing Group.) 